\title{
Summary and Concluding Remarks
}

We present here a personal summary of some of the points that were made during the discussions at the Lake Louise Symposium on "Disorders of Muscle Tone", February 6-8, 1987. It would be invidious and probably impossible to identify the most important issues arising from the quite lengthy interactions between the speakers and their questioners. We wish to stress here that the following account derives from recall which is flawed by the interests and biases that we cannot exclude from our personal memories of the symposium. In addition to commenting on points of interest, we have attempted to include specific progress reports on concepts or controversies debated in the first Canadian Conference on Neurodegenerative Diseases held in Montebello, Quebec, in 1983.

The first day consisted of papers dealing with the basic neuroscience of motor control and its disturbance in disease. $\mathrm{McGeer}$ reported on a new method for identifying regional degeneration in the brain at a microscopic level. He employed HLA-DR staining to demonstrate phagocytotic cells, as an index of neuronal demise. The application of this technique was discussed; its potential value seems to be considerable. Phillips reviewed the nature of the cognitive deficit associated with several motor disorders. This stimulated considerable discussion on the separation of declarative memory (knowing what) versus procedural memory (knowing how), the latter being associated with striatal disease. Lee analyzed the physiological role of the basal ganglia, and his paper provoked discussion on the notion of motor function being processed by multiple neuronal networks operating simultaneously, an operational organization termed "parallel processing'" by computer programmers.

The pharmacology of receptors in the basal ganglia was reviewed, first by Guttman and then by Beaulieu. Since the Montebello meeting, the classification of dopamine receptors in the D1 and D2 types has become firmly accepted. While the D2 receptor is clearly important in Parkinson's disease, recent evidence suggests that the $\mathrm{D} 1$ receptor may facilitate the D2. It was agreed in the discussion that more selective D1 agonists and antagonists were needed to elucidate this possibility, which has substantial implications for the design of new antiparkinson therapy.

In the subsequent discussion, Duvoisin made a plea for uniformity in the use of the general term "parkinsonism" and the specific designation "Parkinson's disease" (i.e. idiopathic parkinsonism) and the need to maintain a distinction between the two. There followed a series of papers on neuroepidemiology and neurotoxins. Some of the topics addressed on the first day were reappraised in more extensive discussion on the last day. In order to present a coherent story we will unite these discussions, and we shall take the liberty of reviewing briefly, the history of the ALS-PD complex of Guam, a disease that may prove to have profound implications for our understanding of neurodegenerative disorders of late life, such as Parkinson's disease, Alzheimer's disease, amyotrophic lateral sclerosis, and progressive supranuclear palsy. The relevant papers in this issue by Spencer and Steele provide much more detail.
Following the Second World War there was considerable interest in the ALS-PD complex of Guam. Early findings of a high familial aggregation initially led investigators to postulate that the disease might have a genetic basis. 'However, careful family pedigrees did not reveal a pattern consistent with mendelian inheritance. Furthermore, the geographic distribution of cases and the occurrence of the disease among non-Chamorros, suggested that an environmental factor was more likely. ${ }^{2.3}$ Epidemiological studies led Whiting, ${ }^{4}$ Kurland and Mulder to suggest that the disorder might stem from the ingestion of flour made from a cycad seed that was prevalent on Guam. Subsequent surveys ${ }^{5,6.7}$ have indicated that the ALS-PD complex of Guam is disappearing as the island becomes westernized, providing further support for an environmental origin.

In 1982, Spencer drew attention to a possible link between lathyrism (a toxic pyramidal disorder) and the ALS-PD complex of Guam. ${ }^{8}$ After studying lathyrism in Asia, modelling the disease in primates, and demonstrating the chronic neurotoxicity of beta- $\mathrm{N}$-oxalylamino-L-alanine (present in the culpable Lathyrus seed), he went on to show that beta-N-methylamino-Lalanine (isolated from Cycas circinalis by Vega and Bell ${ }^{9}$ ) elicited a motoneuron and extrapyramidal disorder reminiscent of the Guam disease. ${ }^{10}$ At Lake Louise, Spencer and Steele noted that the cycad hypothesis was consistent with the current epidemiological knowledge.

In the discussion, Steele, Calne and Schoenberg drew attention to the clinical and pathological similarity between the ALS-PD complex of Guam and progressive supranuclear palsy, a condition first described by Steele et al in 1963. " Duvoisin noted that Kurland had previously commented on the resemblance between the ALS-PD complex of Guam and progressive supranuclear palsy.

This was the first occasion at which Spencer, Steele, Schoenberg and Calne discussed their views together; notable was the remarkable involvement of Steele in the report on progressive supranuclear palsy in Toronto in 1963, and his presence in Guam when the cycad hypothesis re-emerged.

An intriguing aspect of the ALS-PD complex of Guam is documentation of several decades of latency between exposure to the putative agent and appearance of disease. Calne's proposal that age-related localized attrition of neurons might be relevant led to the expression of strong views from the audience, both for and against this hypothesis.

MPTP toxicity was addressed in several papers. Chemicallyrelated toxins were discussed by Heikkila. Crossman described the important finding of lesions outside the substantia nigra, after MPTP, in contrast to the earlier reports in Montebello. Crossman's work strengthens the analogy between MPTP toxicity and Parkinson's disease.

This relationship was further supported by observations reported by Bédard in the discussion. Drug-induced dyskinesia occurred in MPTP-treated monkeys under circumstances identical to those that cause it in Parkinson's disease. Heyes then described studies on quinolinic acid, another neurotoxin of 
particular interest because it is produced endogenously in the nervous system.

The following day was devoted to therapy. In the discussion following the papers of Bouchard, Gauthier, Lieberman and Riopelle, there was growing acceptance of an approach whereby levodopa and bromcriptine are used together in submaximal dosage at a relatively early stage of Parkinson's disease. The concept of treatment designed to slow down the underlying pathology was discussed after Grimes' paper. Following the reports by Lang and Obeso, there was an exchange of views on new drugs and novel routes of administration.

On the last day, there were papers on spasticity and multiple sclerosis. Markham initially bridged the borderland between pyradmidal and extrapyramidal systems by discussing the role of vestibular pathways in relation to each of the major motor organizations. Considerable interest emerged from Paty's demonstration of demyelinating lesions appearing and disappearing from magnetic resonance imaging (MRI) scans, with clinical manifestations only becoming manifest if an "eloquent" portion of the central nervous system was involved. Ashby's and Eisen's review of methods for measuring spasticity and rigidity led to discussion of the similar problems faced by investigators studying pyramidal and extrapyramidal disease, who need to quantify clinical deficits, physiological disturbances, and abnormalities detected by PET and MRI imaging.

Martin and Garnett presented papers that generated some controversy on the merits and limitations of employing carbidopa in the conduct of fluorodopa positron emission tomography (PET) scans. There was also disagreement on the optimal analytic technique for studying age-related impairment of the nigrostriatal pathway, in health and disease.

In the final session, Tsui argued that injection of botulinum toxin was the best treatment for most patients with dystonic torticollis, though more experience is required to establish long-term safety and efficacy. Tatton raised the interesting possibility that botulinum toxin might be transported up nerves to act centrally by decreasing the physiological abnormality of excessive central excitability described by Marsden. After Klawans' paper on Huntington's disease, there were conflicting views on the practical application of preclinical tests for detecting this disorder. Following Jankovic's paper, diverse views were expressed on the prognosis of chronic multiple tic, and there was widespread agreement that the term "Tourette's Syndrome" led to semantic confusion and was probably best abandoned. It also emerged from the discussion that many found tetrabenazine the drug of choice in those cases of chronic multiple tic that really needed pharmacotherapy. The meeting ended with a paper by Duvoisin on progressive supranuclear palsy that led back to discussion on the ALS-PD complex of Guam.

In summary, this symposium identified many areas in which agreement was emerging, but controversies were also evident, and sufficient to provide food for thought for those who might plan a third Canadian Conference on Neurodegenerative Diseases.

Finally, Schoenberg urged us to exercise caution in swallowing new concepts, not backed by facts, while Spencer and Steele argued that perhaps we should be examining what we swallow in a more literal sense.

Donald B. Calne

Bruce S. Schoenberg

\section{REFERENCES}

I. Kurland LT and Mulder DW. Epidemiologic investigations of amyotrophic lateral sclerosis. 2. Familial aggregation indicative of dominant inheritance. Neurology 1955: 5: 182-196.

2. Kurland LT. Introductory remarks. Proceedings of the 3 rd Conference on the toxicity of cycads. Fed Proc 1964; 23: 1337-1339.

3. Reed D. Plato C. Elizan T. et al. The amyotrophic lateral sclerosis/parkinsonism-dementia complex: A ten-year follow-up on Guam. Am J of Epid 1966: 83, 1: 54-73.

4. Whiting MG. Toxicity of Cycads. Econ Bot 1963: 17: $271-302$.

5. Reed DM, Brody JA. Amyotrophic lateral sclerosis and parkinsonismdementia on Guam. 1945-1972. 1. Descriptive epidemiology. Am J of Epid 1975: 101, 4: 287-301.

6. Garruto RM. Yanagihara R. Gajdusek DC. Disappearance of highincidence amyotrophic lateral sclerosis and parkinsonism-dementia on Guam. Neurol 1985: 35: 193-198.

7. Rodgers-Johnson P, Garruto RM, Yanagihara R, et al. Amyotrophic lateral sclerosis and parkinsonism-dementia on Guam: A 30 year old evaluation of clinical and neuropathologic trends. Neurol 1986; 36: 7-13.

8. Spencer PS, Schaumburg HH. The pathogenesis of motor neuron disease: Perspectives from neurotoxicology. In: Human Motor Neuron Disease, Rowland LP. Advances in Neurology, vol 36. Raven Press, N.Y.. 1982: 249.

9. Vega A, Bell EA. $\alpha$-Amino- $\beta$-methylaminopropionic acid, a new amino acid from seeds of Cycas circinalis. Phytochemistry (Oxf) 1967; 6: 759-762

10. Spencer PS, Hugon J, Ludolph A. et al. Discovery and partial characterization of primate motor-system toxins. In: Bock G. O'Connor M. eds., Selective Neuronal Death. Ciba Foundation Symposium. Wiley Interscience 1987: 221-238.

11. Richardson JC. Steele JC. Olszewski J. Supranuclear opthalmoplegia pseudolbar palsy, nuchal dystonia and dementia: A clinical report on eight cases of "heterogeneous degeneration". Trans Amer Neurol Assoc 1963: 88: 25-29. 